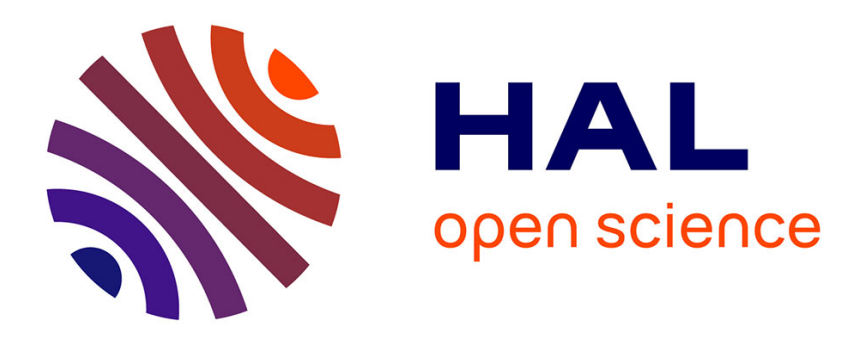

\title{
On measures of nonlinearity effects for uncertain dynamical systems - Application to a vibro-impact system
}

R. Sampaio, Christian Soize

\section{- To cite this version:}

R. Sampaio, Christian Soize. On measures of nonlinearity effects for uncertain dynamical systems Application to a vibro-impact system. Journal of Sound and Vibration, 2007, 303 (3-5), pp.659-674. 10.1016/j.jsv.2007.01.033 . hal-00686149

\section{HAL Id: hal-00686149 \\ https://hal.science/hal-00686149}

Submitted on 7 Apr 2012

HAL is a multi-disciplinary open access archive for the deposit and dissemination of scientific research documents, whether they are published or not. The documents may come from teaching and research institutions in France or abroad, or from public or private research centers.
L'archive ouverte pluridisciplinaire HAL, est destinée au dépôt et à la diffusion de documents scientifiques de niveau recherche, publiés ou non, émanant des établissements d'enseignement et de recherche français ou étrangers, des laboratoires publics ou privés. 


\title{
On measures of non-linearity effects for uncertain dynamical systems - Application to a vibro-impact system
}

\author{
Rubens Sampaio* \\ Pontifícia Universidade Católica do Rio de Janeiro, Departamento de Engenharia \\ Mecânica, Rua Marquês de São Vicente 225, Gávea, 22453-900, Rio de Janeiro, \\ RJ, Brazil \\ Christian Soize \\ Universite de Marne la Vallee, Laboratoire de Mecanique \\ 5 Bd Descartes, Champs sur Marne, 77454 Marne la Vallee, France
}

\begin{abstract}
This paper studies the transient dynamics of a linear dynamical system with elastic barriers excited by a deterministic transient force whose Fourier Transform has a bounded frequency narrow band. The system is then non-linear. In order to measure the degree of non-linearity of the system, one looks for the mechanical energy transferred outside the frequency band of excitation as a function of the parameter $\eta$ defined by $\varepsilon / a$, in which $\varepsilon$ is the size of the barrier gap and $a$ is the amplitude of the excitation force. The mechanical energy transferred outside the frequency band of excitation can potentially be a source of excitation for other subsystems. Consequently, a quantification of this energy transfer is important for the understanding of the non-linear dynamical system. In addition, it is well known that this type of non-linear dynamical system is very sensitive to uncertainties. For this reason one studies the system as being deterministic, and also stochastic in order to take into account random uncertainties. The proposed analysis is then applied to a Timoshenko beam having its motion constrained by a symmetric elastic barrier at its free end. In particular, one shows the confidence region of the random mechanical energy transferred outside the excitation band as a function of $\eta$ for several levels
\end{abstract}


of model and data uncertainties. This type of results allows the robustness of the predictions to be analyzed with respect to model and data uncertainties.

Key words: Non-linear Dynamics, Vibro-impacts, System Uncertainties

\section{Introduction}

The non-linear dynamics of linear dynamical systems with barriers inducing impacts has received considerable attention in the last two decades. Although there are some engineering systems where impacts are part of the project, most of the time this phenomenon is related to wear, fatigue and noise as, for example, in the case of gear boxes. The interest in vibro-impact systems arises due to their intrinsic non-linear characteristic which prevents their study through more traditional methods such as modal analysis. Actually, systems of this kind have an extremely complex dynamic behaviour, sometimes even chaotic. Therefore, they are normally studied with bifurcation diagrams and Poincaré maps. However, most of the vibro-impact systems investigated so far consists of simple ones with a single degree of freedom. It is expected that the flexibility of a structure will play an important role in its impact response, specially through the excitations of many of its degrees of freedom. Also one expects some exchange of energy among modes due to impacts. A lot of works have been published concerning one single degree of freedom and multi-degrees of freedom deterministic systems excited by deterministic harmonic signals or by narrow- or wide-band stochastic processes (see for instance Babitsky and Birkett [1]). Some works were also published concerning the identification of restoring force non-linearities from system response to white noise excitation (see for instance Dimentberg and Sokolov [2] for single degree-of-freedom systems). A review of such works can be found in the recent paper by Dimentberg and Iourtchenko [3]. It should be noted that deterministic continuous systems with impacts have received less attention probably due to the difficulties of

\footnotetext{
* Corresponding author. Tel: +55 213114 1172, Fax: +55 2133141165

Email addresses: rsampaio@mec.puc-rio.br (Rubens Sampaio), soize@univ-mlv.fr (Christian Soize).
} 
such non-linear dynamical problems which are very hard to analyze with analytical tools or with numerical methods. However, some recent representative works of this type can be found in Refs. [4-8].

Some of the features of this work are:

- It is not about a single, nor multiple, degree of freedom system, but deals with a continuous system. Nevertheless, in order to simplify the presentation and also to show that the methodology applies to a general dynamical system, we start with a discretization of the continuous system, say by using the finite element method.

- The excitation is neither narrow- nor broad-band stochastic process (including white noise modelling) nor deterministic harmonic signal. In this paper the excitation will be modelled by a deterministic narrow-band signal. This choice is important because it gives some robustness to the excitation. One centers the band around one of the natural frequencies of the linear system (without impact) and the width of the band is chosen in order to allow modifications of the system to be taken into account (non-linearities and uncertainties).

- It deals with the deterministic and also stochastic modelling of the continuous system. The stochastic aspects being induced by the uncertainties in the data and in the model (the matrices that represent the linear continuous system are random).

- Measures of non-linearities are proposed. In order to analyze the degree of non-linearity of the system, one looks for the mechanical energy transferred outside the frequency band of excitation as a function of the parameter $\eta$, defined by $\varepsilon / a$, in which $\varepsilon$ is the size of the barrier gap and $a$ is the amplitude of the excitation force. When $\varepsilon$ is zero or infinity, there are no impacts. When it is between this two bounds the continuous system-barrier behaves non-linearly for amplitude $a$ sufficiently high. It turns out that the non-linearity depends on $\eta$. The interest of measuring the amount of energy that is transferred outside the band of excitation is to evaluate the dangerous consequences like exciting sensitive subsystems whose lowest eigenfrequency is outside the band of excitation.

- Stochastic systems are considered in order to evaluate the robustness of 
the numerical prediction of the energy transferred with respect to data and model uncertainties.

This paper is divided into four parts. Section 2 is devoted to the modelling and analysis of the deterministic non-linear dynamical system. In Section 3 one presents the stochastic modelling of the system in order to take into account data and model uncertainties. Section 4 deals with numerical applications. We take a Timoshenko beam with an elastic barrier. Finally, general analysis and conclusions are presented in Section 5.

\section{Modelling and analysis of the deterministic non-linear dynamical system}

In this section one presents the mean model of the dynamical system with excitation, the reduced mean model obtained by using the elastic modes of the linear mean dynamical system and, finally, one describes the different energies one needs to analyze the energy transferred outside the excitation band.

\subsection{Finite element model of the mean non-linear dynamical system}

The main interest of the paper is to study a linear continuous system with elastic barriers that induce through impact non-linearities. However the methodology one presents is general and can be applied to a larger class of problems, as for example those related to a linear system interacting with a subsystem that originates non-linearities, as the case of an elastic barrier. In order to focus in the methodology one starts with a finite dimensional system that could be the result of a discretization process. This system, referred as the mean model, is described by the following matrix equation in $\mathbb{R}^{m}$,

$$
[\mathbb{M}] \ddot{\mathbf{y}}(t)+[\mathbb{D}] \dot{\mathbf{y}}(t)+[\mathbb{K}] \mathbf{y}(t)+\mathbf{f}_{\mathrm{NL}}(\mathbf{y}(t), \dot{\mathbf{y}}(t))=\mathbf{f}(t)
$$

where $[\mathbb{M}],[\mathbb{D}],[\mathbb{K}]$ are the mass, damping, and stiffness matrices, that are supposed to be symmetric and positive-definite real matrices, $\mathbf{y}(t)$ is the dis- 
placement vector, $\mathbf{f}_{\mathrm{NL}}(\mathbf{y}(t), \dot{\mathbf{y}}(t))$ describes the non-linear vector forces, $\mathbf{f}(t)$ the applied vector load. The non-linear mapping $(\mathbf{y}, \mathbf{z}) \mapsto \mathbf{f}_{\mathrm{NL}}(\mathbf{y}, \mathbf{z})$ is assumed to be such that $\mathbf{f}_{\mathrm{NL}}(0,0)=0$. The vector load $\mathbf{f}(t)$ is written as

$$
\mathbf{f}(t)=\operatorname{ag}(t) \mathbf{f}_{0}
$$

in which $a$ is the amplitude and $\mathbf{f}_{0}$ is a normalized vector describing the position of the applied forces. The impulse $t \mapsto g(t)$ is a square integrable real-valued function on $\mathbb{R}$ whose Fourier Transform $\omega \mapsto \widehat{g}(\omega)=\int_{\mathbb{R}} e^{-i \omega t} g(t) d t$ has a bounded support $\underline{B}_{2} \cup B_{2}$ with

$$
B_{2}=\left[\omega_{\min }, \omega_{\max }\right] \quad, \quad \underline{B}_{2}=\left[-\omega_{\max },-\omega_{\min }\right] .
$$

The notation $B_{2}$ will be explained in Section 2.3. In addition it is assumed that $\max _{\omega \in B_{2}}|\widehat{g}(\omega)|=1$.

\subsection{Reduced mean model}

Let $\left\{\phi_{1}, \ldots, \phi_{m}\right\}$ be an algebraic basis of $\mathbb{R}^{m}$. The reduced mean model of the dynamic system whose mean finite element model is defined by Eq. (1) is obtained by projection of Eq. (1) on the subspace $V_{n}$ of $\mathbb{R}^{m}$ spanned by $\left\{\phi_{1}, \ldots, \phi_{n}\right\}$ with $n \ll m$. Let $\left[\Phi_{n}\right]$ be the $(m \times n)$ real matrix whose columns are the vectors $\left\{\phi_{1}, \ldots, \phi_{n}\right\}$. The generalized applied force $\mathbf{F}^{n}(t)$ is an $\mathbb{R}^{n}$ vector such that $\mathbf{F}^{n}(t)=\left[\Phi_{n}\right]^{T} \mathbf{f}(t)$. The generalized mass, damping, and stiffness matrices, $\left[\underline{M}_{n}\right],\left[\underline{D}_{n}\right]$, and $\left[\underline{K}_{n}\right]$, are positive-definite symmetric $(n \times n)$ real matrices such that $\left[\underline{M}_{n}\right]=\left[\Phi_{n}\right]^{T}[\mathbb{M}]\left[\Phi_{n}\right],\left[\underline{D}_{n}\right]=\left[\Phi_{n}\right]^{T}[\mathbb{D}]\left[\Phi_{n}\right]$, and $\left[\underline{K}_{n}\right]=\left[\Phi_{n}\right]^{T}[\mathbb{K}]\left[\Phi_{n}\right]$. Consequently, the reduced mean model of the nonlinear dynamic system, written as the projection $\mathbf{y}^{n}$ of $\mathbf{y}$ on $V_{n}$, can be written as

$$
\mathbf{y}^{n}(t)=\left[\Phi_{n}\right] \mathbf{q}^{n}(t),
$$

in which the vector $\mathbf{q}^{n}(t) \in \mathbb{R}^{n}$ of the generalized coordinates verifies the mean non-linear differential equation,

$$
\left[\underline{M}_{n}\right] \ddot{\mathbf{q}}^{n}(t)+\left[\underline{D}_{n}\right] \dot{\mathbf{q}}^{n}(t)+\left[\underline{K}_{n}\right] \mathbf{q}^{n}(t)+\mathbf{F}_{\mathrm{NL}}^{n}\left(\mathbf{q}^{n}(t), \dot{\mathbf{q}}^{n}(t)\right)=\mathbf{F}^{n}(t)
$$


where, for all $\mathbf{q}$ and $\mathbf{p}$ in $\mathbb{R}^{n}$,

$$
\mathbf{F}_{\mathrm{NL}}^{n}(\mathbf{q}, \mathbf{p})=\left[\Phi_{n}\right]^{T} \mathbf{f}_{\mathrm{NL}}\left(\left[\Phi_{n}\right] \mathbf{q},\left[\Phi_{n}\right] \mathbf{p}\right)
$$

\subsection{Quantification of the transferred energies outside the excitation band}

The objective of this section is to quantify the mechanical energy transferred outside the excitation band. It is assumed that Eq. (1) has a unique solution $t \mapsto \mathbf{y}(t)$ such that $\mathbf{y}$ and $\dot{\mathbf{y}}$ are square integrable vector-valued functions on $\mathbb{R}$. An approximation of this solution is computed using the reduced mean model defined by Eqs. (4)-(6). The positive frequency band $\mathbb{R}^{+}=[0,+\infty[$ is then written as

$$
\mathbb{R}^{+}=\left[0,+\infty\left[=B_{1} \cup B_{2} \cup B_{3},\right.\right.
$$

in which $B_{1}=\left[0, \omega_{\min }\left[\right.\right.$ and $\left.B_{3}=\right] \omega_{\max },+\infty\left[\right.$. The sets $B_{1}$ and $B_{3}$ are the bands outside the frequency band of excitation $B_{2}$. The total mechanical energy, denoted by $\widetilde{e}$, of the non-linear dynamical system corresponding to the solution mentioned above is written as,

$$
\widetilde{e}=\int_{\mathbb{R}}\left(\frac{1}{2}<[\mathbb{M}] \dot{\mathbf{y}}(t), \dot{\mathbf{y}}(t)>+\frac{1}{2}<[\mathbb{K}] \mathbf{y}(t), \mathbf{y}(t)>\right) d t
$$

Let $\widehat{\mathbf{y}}(\omega)=\int_{\mathbb{R}} e^{-i \omega t} \mathbf{y}(t) d t$ be the Fourier Transform of $\mathbf{y}$. Using the Parseval formula, Eq. (8) yields

$$
\widetilde{e}=\int_{\mathbb{R}} \underline{h}(\omega) d \omega=2 \int_{\mathbb{R}^{+}} \underline{h}(\omega) d \omega,
$$

in which $\underline{h}(\omega)$ is the density of the mechanical energy in the frequency domain which is written as

$$
\underline{h}(\omega)=\frac{1}{2 \pi}\left\{\frac{1}{2}<\omega^{2}[\mathbb{M}] \widehat{\mathbf{y}}(\omega), \overline{\widehat{\mathbf{y}}(\omega)}>+\frac{1}{2}<[\mathbb{K}] \widehat{\mathbf{y}}(\omega), \overline{\widehat{\mathbf{y}}(\omega)}>\right\} .
$$

From Eqs. (7) and (9), it can deduced that

$$
\widetilde{e}=\widetilde{e}_{1}+\widetilde{e}_{2}+\widetilde{e}_{3}
$$

in which

$$
\widetilde{e}_{j}=2 \int_{B_{j}} \underline{h}(\omega) d \omega \quad, \quad j=1,2,3
$$


The transferred mechanical energy outside the excitation band $B_{2}$ is denoted by $\widetilde{e}_{13}$ which is defined by

$$
\widetilde{e}_{13}=\widetilde{e}_{1}+\widetilde{e}_{3}
$$

Using the reduced mean model defined by Eqs. (4)-(6), the approximation $\underline{h}^{n}(\omega)$ of $\underline{h}(\omega)$ defined by Eq. (10) can be written as

$$
\underline{h}^{n}(\omega)=\frac{1}{2 \pi}\left\{\frac{1}{2}<\omega^{2}\left[\underline{M}_{n}\right] \widehat{\mathbf{q}}^{n}(\omega), \overline{\widehat{\mathbf{q}}^{n}(\omega)}>+\frac{1}{2}<\left[\underline{K}_{n}\right] \widehat{\mathbf{q}}^{n}(\omega), \overline{\widehat{\mathbf{q}}^{n}(\omega)}>\right\},
$$

in which $\widehat{\mathbf{q}}^{n}(\omega)=\int_{\mathbb{R}} e^{-i \omega t} \mathbf{q}^{n}(t) d t$ is the Fourier Transform of $\mathbf{q}^{n}$. The corresponding energies computed with this approximation are denoted by $\widetilde{e}^{n}$, $\widetilde{e}_{1}^{n}, \widetilde{e}_{2}^{n}, \widetilde{e}_{3}^{n}, \widetilde{e}_{13}^{n}$. In order to explore the results in a non-dimensional way one introduces the following parameters,

$$
e_{1}^{n}=\frac{\widetilde{e}_{1}^{n}}{\widetilde{e}^{n}} \quad, \quad e_{2}^{n}=\frac{\widetilde{e}_{2}^{n}}{\widetilde{e}^{n}} \quad, \quad e_{3}^{n}=\frac{\widetilde{e}_{3}^{n}}{\widetilde{e}^{n}} \quad, \quad e_{13}^{n}=\frac{\widetilde{e}_{13}^{n}}{\widetilde{e}^{n}}
$$

Consequentely, one has,

$$
e_{1}^{n}+e_{2}^{n}+e_{3}^{n}=1 \quad, \quad e_{13}^{n}+e_{2}^{n}=1
$$

The $e_{13}^{n}$ represents the percentage of mechanical energy transferred outside the frequency band of the excitation band.

\section{Modelling and analysis of the non-linear dynamical system with random uncertainties}

The first source of uncertainties in this type of problem is due to the mathematicalmechanical modelling process leading to the boundary value problem. This type of uncertainty is structural, and cannot be represented as, simply, the usual variation of parameters $[9,10]$. This uncertainties are called the model uncertainties. Concerning the second source of uncertainties, they come from the parameters such as geometry, material properties, boundary and initial conditions, etc, related to the boundary value problem. The uncertainties in these parameters are called data uncertainties. It is worthwhile to remark that the errors related to the construction of an approximation of the solution 
of the boundary value problem, that have to be controlled in order to meet the specifications of the numerical approximation, are not uncertainties.

For the class of systems one studies the sources of uncertainties are in the data related to the non-linear term and in the data and model related to the linear part.

\subsection{Probabilistic modelling of uncertainties}

From this point one constructs the probability model of uncertainties from the mean reduced model defined by Eqs. (4)-(6). All the random variables are defined in a probability space $(\Theta, \mathcal{F}, \mathcal{P})$

(A) Parametric probabilistic model of data uncertainties for the non-linear term. Usually, data uncertainties are modelled by using parametric probabilistic approach consisting in modelling each uncertain parameter by a random variable whose probability distribution has to be constructed using the available information. The non-linear term $\mathbf{F}_{\mathrm{NL}}^{n}\left(\mathbf{q}^{n}(t), \dot{\mathbf{q}}^{n}(t)\right)$ in Eq. (5) is rewritten as $\mathbf{F}_{\mathrm{NL}}^{n}\left(\mathbf{q}^{n}(t), \dot{\mathbf{q}}^{n}(t) ; \mathbf{s}\right)$ in which $\mathbf{s}$ is an $\mathbb{R}^{\nu}$-vector of uncertain parameters. The probabilistic modelling of vector $\mathbf{s}$ is as a $\mathbb{R}^{\nu}$-valued random variable whose probability distribution on $\mathbb{R}^{\nu}$ is denoted by $P_{\mathbf{S}}(d \mathbf{s})$. The available information for constructing $P_{\mathbf{S}}(d \mathbf{s})$ depends on the nature of the parameters constituting the vector s (for instance, positivity, boundedness of components, etc). When this information is defined the probability distribution can be constructed using the maximum entropy principle with the constraints defined by the available information $[11,13]$.

(B) Non-parametric probabilistic model of model and data uncertainties for the linear part. Model uncertainties cannot be taken into account using the parametric probabilistic approach. A non-parametric probabilistic approach can be used to take into account model uncertainties and data uncertainties $[9,10]$. The principle of construction of such non-parametric probabilistic approach of uncertainties for the linear part of the non-linear dynamical system whose reduced mean model is defined by Eqs. (4)-(6) consists in substituting the generalized mass, damping, and stiffness matrices in Eq. (5) by the ran- 
dom matrices $\left[\mathbf{M}_{\mathbf{n}}\right],\left[\mathbf{D}_{\mathbf{n}}\right]$, and $\left[\mathbf{K}_{\mathbf{n}}\right]$ whose probability distributions have been constructed using the maximum entropy principle with an adapted available information. The explicit form of the probability distributions of the random matrices $\left[\mathbf{M}_{\mathbf{n}}\right],\left[\mathbf{D}_{\mathbf{n}}\right]$, and $\left[\mathbf{K}_{\mathbf{n}}\right]$ are given in Refs. $[9,10]$.

(C) Stochastic reduced model. The stochastic transient response of the nonlinear dynamic system with a non-parametric probabilistic approach of model and data uncertainties is the stochastic process $\mathbf{Y}^{n}(t)$, indexed by $\mathbb{R}$, with values in $\mathbb{R}^{m}$, such that

$$
\mathbf{Y}^{n}(t)=\left[\Phi_{n}\right] \mathbf{Q}^{n}(t)
$$

in which the stochastic process $\mathbf{Q}^{n}$, defined in the probability space $(\Theta, \mathcal{F}, \mathcal{P})$, indexed by $\mathbb{R}$, with values $\mathbb{R}^{n}$, is such that

$$
\begin{aligned}
& {\left[\mathbf{M}_{n}\right] \ddot{\mathbf{Q}}^{n}(t)+\left[\mathbf{D}_{n}\right] \dot{\mathbf{Q}}^{n}(t)+\left[\mathbf{K}_{n}\right] \mathbf{Q}^{n}(t)+} \\
& \mathbf{F}_{N L}^{n}\left(\mathbf{Q}^{n}(t), \dot{\mathbf{Q}}^{n}(t) ; \mathbf{S}\right)=\mathbf{F}^{n}(t) \quad, \quad \forall t \in \mathbb{R} .
\end{aligned}
$$

Let ||$\left|\mathbf{Q}^{n}\right| \mid$ be the norm such that

$$
\left\|\mathbf{Q}^{n}\right\| \|^{2}=\mathcal{E}\left\{\int_{\mathbb{R}}\left\|\mathbf{Q}^{n}(t)\right\|^{2} d t\right\}
$$

in which $\mathcal{E}$ is the mathematical expectation and where $\|\mathbf{u}\|^{2}=u_{1}^{2}+\ldots+u_{n}^{2}$ is the square of the Euclidean norm of $\mathbf{u}$ in $\mathbb{R}^{n}$. It is assumed that the non-linear term is such that Eq. (18) has a unique second-order mean-square solution such that

$$
\left\|\left|\mathbf{Q}^{n}\right|\left|<+\infty \quad, \quad \| \dot{\mathbf{Q}}^{n}\right|||<+\infty .\right.
$$

3.2 Probabilistic quantification of the transferred energies outside the excitation band for the uncertain system

The objective of this section is to adapt Section 2.3 to the reduced stochastic system defined by Eqs. (17) and (18). The random total mechanical energy associated with $\widetilde{e}^{n}$ is denoted by $\widetilde{E}^{n}$ and is such that

$$
\widetilde{E}^{n}=\int_{\mathbb{R}}\left(\frac{1}{2}<[\mathbb{M}] \dot{\mathbf{Y}}^{n}(t), \dot{\mathbf{Y}}^{n}(t)>+\frac{1}{2}<[\mathbb{K}] \mathbf{Y}^{n}(t), \mathbf{Y}^{n}(t)>\right) d t
$$


The density of the random mechanical energy in the frequency domain associated with $\underline{h}^{n}(\omega)$ defined by Eq. (14) is denoted by $H^{n}(\omega)$ and can be written as

$$
H^{n}(\omega)=\frac{1}{2 \pi}\left\{\frac{1}{2}<\omega^{2}\left[\underline{M}_{n}\right] \widehat{\mathbf{Q}}^{n}(\omega), \overline{\widehat{\mathbf{Q}}^{n}(\omega)}>+\frac{1}{2}<\left[\underline{K}_{n}\right] \widehat{\mathbf{Q}}^{n}(\omega), \overline{\widehat{\mathbf{Q}}^{n}(\omega)}>\right\}
$$

in which $\widehat{\mathbf{Q}}^{n}(\omega)=\int_{\mathbb{R}} e^{-i \omega t} \mathbf{Q}^{n}(t) d t$ is the Fourier Transform of $\mathbf{Q}^{n}$.

Let $H_{\mathrm{dB}}^{n}(\omega)$ be the density of the random mechanical energy in $\mathrm{dB}$ normalized with respect to the total mechanical energy $\widetilde{e}_{\text {lin }}$ of the linear mean system. One then has

$$
H_{\mathrm{dB}}^{n}(\omega)=\log _{10}\left(H^{n}(\omega) / \widetilde{e}_{\mathrm{lin}}\right) .
$$

Let $\widetilde{E}_{1}^{n}, \widetilde{E}_{2}^{n}, \widetilde{E}_{3}^{n}$ and $\widetilde{E}_{13}^{n}$ be the random energies associated with $\widetilde{e}_{1}^{n}, \widetilde{e}_{2}^{n}, \widetilde{e}_{3}^{n}$ and $\widetilde{e}_{13}^{n}$ such that

$$
\widetilde{E}_{j}^{n}=2 \int_{B_{j}} H^{n}(\omega) d \omega \quad, \quad j=1,2,3 \quad, \quad \widetilde{E}_{13}^{n}=\widetilde{E}_{1}^{n}+\widetilde{E}_{3}^{n} .
$$

Similarly to Section 2.3, this random energies are normalized as follows

$$
E_{1}^{n}=\frac{\widetilde{E}_{1}^{n}}{\widetilde{E}^{n}} \quad, \quad E_{2}^{n}=\frac{\widetilde{E}_{2}^{n}}{\widetilde{E}^{n}} \quad, \quad E_{3}^{n}=\frac{\widetilde{E}_{3}^{n}}{\widetilde{E}^{n}} \quad, \quad E_{13}^{n}=\frac{\widetilde{E}_{13}^{n}}{\widetilde{E}^{n}} .
$$

Consequentely, one has the random equations,

$$
E_{1}^{n}+E_{2}^{n}+E_{3}^{n}=1 \quad, \quad E_{13}^{n}+E_{2}^{n}=1 .
$$

The $E_{13}^{n}$ represents the percentage of the random mechanical energy transferred outside the frequency band of the excitation band.

\subsection{Stochastic solver and convergence}

In this section one introduces the stochastic solver that is used and one analyses the stochastic convergence. The Monte Carlo numerical simulation and mathematical statistics are used for solving the stochastic equations defined by Eqs. (17) and (18). Let $\mathbf{S}(\theta)$ and $\left[\mathbf{M}_{n}(\theta)\right],\left[\mathbf{D}_{n}(\theta)\right],\left[\mathbf{K}_{n}(\theta)\right]$ be independent realizations of the random variable $\mathbf{S}$ and the random matrices $\left[\mathbf{M}_{n}\right],\left[\mathbf{D}_{n}\right]$, $\left[\mathbf{K}_{n}\right]$, for $\theta \in \Theta$. 
(A) Construction of realizations of random variable $\mathbf{S}$. Each realization $\mathbf{S}(\theta)$ of random variable $\mathbf{S}$ is usually constructed using random generator associated with the probability distribution $P_{\mathbf{S}}(d \mathbf{s})$. Because the generation is standard it will not be detailed here.

(B) Construction of realizations of random matrix variables $\left[\mathbf{M}_{n}\right],\left[\mathbf{D}_{n}\right],\left[\mathbf{K}_{n}\right]$. Let $\left[\mathbf{A}_{n}\right]$ be any of the three random matrices above and let $\left[\underline{\mathbf{A}}_{n}\right]$ be its mean value which is a positive-definite matrix. Its Cholesky factorization yields $\left[\underline{\mathbf{A}}_{n}\right]=\left[\underline{L}_{n}\right]^{T}\left[\underline{L}_{n}\right]$. Each realization $\left[\mathbf{A}_{n}(\theta)\right]$ can be generated using the following algebraic representation $[9,10]$,

$$
\left[\mathbf{A}_{n}\right]=\left[\underline{L}_{n}\right]^{T}\left[\mathbf{G}_{n}\right]\left[\underline{L}_{n}\right]
$$

In which the positive-definite random matrix $\left[\mathbf{G}_{n}\right]$ is written as

$$
\left[\mathbf{G}_{n}\right]=\left[\mathbf{L}_{n}\right]^{T}\left[\mathbf{L}_{n}\right]
$$

In Eq. (27), $\left[\mathbf{L}_{n}\right]$ is an upper triangular random matrix with values in $\mathbb{M}_{n}(\mathbb{R})$ such that:

(1) The random variables $\left\{\left[\mathbf{L}_{n}\right]_{j j^{\prime}}, j \leq j^{\prime}\right\}$ are independent.

(2) For $j<j^{\prime}$, the real-valued random variable $\left[\mathbf{L}_{n}\right]_{j j^{\prime}}$ can be written as $\left[\mathbf{L}_{n}\right]_{j j^{\prime}}=\sigma_{n} U_{j j^{\prime}}$ in which $\sigma_{n}=\delta(n+1)^{-1 / 2}$ and where $U_{j j^{\prime}}$ is a real-valued Gaussian random variable with zero mean and variance equal to 1 .

(3) For $j=j^{\prime}$, the positive-valued random variable $\left[\mathbf{L}_{n}\right]_{j j}$ can be written as $\left[\mathbf{L}_{n}\right]_{j j}=\sigma_{n} \sqrt{2 V_{j}}$ in which $\sigma_{n}$ is defined above and where $V_{j}$ is a positive-valued gamma random variable whose probability density function $p_{V_{j}}(v)$ with respect to $d v$ is written as

$$
p_{V_{j}}(v)=\mathbf{1}_{\mathbb{R}^{+}}(v) \frac{1}{\Gamma\left(\frac{n+1}{2 \delta^{2}}+\frac{1-j}{2}\right)} v^{\frac{n+1}{2 \delta^{2}}-\frac{1+j}{2}} e^{-v},
$$

in which $\mathbf{1}_{\mathbb{R}^{+}}(v)=1$ if $v \in \mathbb{R}^{+}$and $=0$ if not, and where $\Gamma$ is the usual Gamma function. This algebraic representation exhibits $\delta$ which is the positive parameter allowing the dispersion of the random matrix $\left[\mathbf{A}_{n}\right]$ to be controlled. This parameter has to be given for each random matrix and controls the level of uncertainties. In special it controls the uncertainties of mass, damping or 
stiffness of the linear continuous system of the non-linear dynamical system.

(C) Construction of realizations of the solution of the stochastic reduced system. The realization $\mathbf{Y}^{n}(t, \theta)$ for $\theta \in \Theta$ of $\mathbf{Y}^{n}(t)$ defined by Eq. (23) is given by

$$
\mathbf{Y}^{n}(t, \theta)=\left[\Phi_{n}\right] \mathbf{Q}^{n}(t, \theta)
$$

in which the realization $\left\{\mathbf{Q}^{n}(t, \theta), t \in \mathbb{R}\right\}$ of the stochastic process $\left\{\mathbf{Q}^{n}(t), t \in\right.$ $\mathbb{R}\}$, is the solution of the following deterministic non-linear reduced equation,

$$
\begin{aligned}
& {\left[\mathbf{M}_{n}(\theta)\right] \ddot{\mathbf{Q}}^{n}(t, \theta)+\left[\mathbf{D}_{n}(\theta)\right] \dot{\mathbf{Q}}^{n}(t, \theta)+\left[\mathbf{K}_{n}(\theta)\right] \mathbf{Q}^{n}(t, \theta)+} \\
& \mathbf{F}_{N L}^{n}\left(\mathbf{Q}^{n}(t, \theta), \dot{\mathbf{Q}}^{n}(t, \theta) ; \mathbf{S}(\theta)\right)=\mathbf{F}^{n}(t, \theta) \quad, \quad \forall t \in \mathbb{R} .
\end{aligned}
$$

This equation is solved by using an implicit unconditionnally stable scheme such as Newmark algorithm. At each time step, the non-linear algebraic equation coming from the scheme is solved by iteration.

(D) Stochastic convergence The mean-square convergence of the second-order stochastic solution of Eq. (18) with respect to dimension $n$ of the stochastic reduced model and to the number $n_{s}$ of realizations used in the Monte Carlo numerical simulations is controlled by the norm $\|\left|\mathbf{Q}^{n}\right|||$ defined by Eq. (19). Using the usual estimation of the mathematical expectation operator $\mathcal{E}$, convergence with respect to $n$ and $n_{s}$ is studied by constructing the function $\left(n_{s}, n\right) \mapsto \operatorname{conv}\left(n_{s}, n\right)$ defined by

$$
\operatorname{conv}\left(n_{s}, n\right)=\left\{\frac{1}{n_{s}} \sum_{k=1}^{n_{s}} \int_{\mathbb{R}}\left\|\mathbf{Q}^{n}\left(t, \theta_{k}\right)\right\|^{2} d t\right\}^{1 / 2},
$$

in which $\mathbf{Q}^{n}\left(t, \theta_{1}\right), \ldots, \mathbf{Q}^{n}\left(t, \theta_{n_{s}}\right)$ are $n_{s}$ independent realizations of $\mathbf{Q}^{n}(t)$.

(E) Statistical estimations of the random energies. One is interested in constructing statistical estimations for the stochastic process $\left\{H^{n}(\omega), \omega \in \mathbb{R}\right\}$ defined by Eq. (22) and for the random variables $E_{1}^{n}, E_{2}^{n}, E_{3}^{n}, E_{13}^{n}$ defined by Eq. (24), whose realizations are directly deduced from the realizations of $\mathbf{Q}^{\mathbf{n}}$. Let $X$ be the positive-valued random variable representing either $H^{n}(\omega)$ for $\omega$ fixed in $\mathbb{R}$ or any of the random variables $E_{1}^{n}, E_{2}^{n}, E_{3}^{n}, E_{13}^{n}$. The mean value 
$m_{X}=\mathcal{E}\{X\}$ is estimated by

$$
\widetilde{m}_{X}=\frac{1}{n_{s}} \sum_{k=1}^{n_{s}} X\left(\theta_{k}\right)
$$

in which $X\left(\theta_{1}\right), \ldots, X\left(\theta_{n_{s}}\right)$ are $n_{s}$ independent realizations of $X$. The confidence region of random variable $X$ is constructed by using the quantiles. Let $F_{X}$ be the cumulative distribution function (continuous from the right) of random variable $X$ such that $F_{X}(x)=P(X \leq x)$. For $0<p<1$, the $p$ th quantile (or fractile) of $F_{X}$ is defined by

$$
\zeta(p)=\inf \left\{x: F_{X}(x) \geq p\right\}
$$

Then the upper envelope $x^{+}$and the lower envelope $x^{-}$of the confidence region with probability level $P_{c}$ are defined by

$$
x^{+}=\zeta\left(\left(1+P_{c}\right) / 2\right) \quad, \quad x^{-}=\zeta\left(\left(1-P_{c}\right) / 2\right) .
$$

The estimations of $x^{+}$and $x^{-}$are performed by using the sample quantiles. Let $x_{1}=X\left(\theta_{1}\right), \ldots, x_{n_{s}}=X\left(\theta_{n_{s}}\right)$. Let $\widetilde{x}_{1}<\ldots<\widetilde{x}_{n_{s}}$ be the order statistics associated with $x_{1}<\ldots<x_{n_{s}}$. Therefore, one has the following estimations,

$$
\begin{aligned}
& x^{+} \simeq \widetilde{x}_{j^{+}} \quad, \quad j^{+}=\operatorname{fix}\left(n_{s}\left(1+P_{c}\right) / 2\right), \\
& x^{-} \simeq \widetilde{x}_{j^{-}} \quad, \quad j^{-}=\operatorname{fix}\left(n_{s}\left(1-P_{c}\right) / 2\right),
\end{aligned}
$$

in which $\operatorname{fix}(z)$ is the integer part of the real number $z$.

\section{Application to a Timoshenko beam with an elastic barrier}

This section deals with the application of the theory developed in the previous sections. The linear part of the continuous system is a Timoshenko beam with added dissipation. The non-linear force is due to a symmetrical linear elastic barrier. 


\subsection{Description of the non-linear elastic dynamical system}

The geometrical properties of the beam are: length $1 \mathrm{~m}$, width $0.1 \mathrm{~m}$, height $0.1 \mathrm{~m}$. The boundary conditions are of a cantilever beam, with the free end having its motion limited by an elastic barrier distant of $\varepsilon$, in both sides of the beam. The gap $\varepsilon$ is considered as a parameter. The beam is homogeneous, isotropic, whose material properties are: density $7500 \mathrm{~kg} / \mathrm{m}^{3}$, Young' s modulus $2.1 \times 10^{10} \mathrm{~N} / \mathrm{m}^{2}$, Poisson's coefficient 0.3 , shearing correction factor $5 / 6$. The damping model is introduced by the model damping rate which is 0.02 for the first three modes, 0.01 for the fourth mode and 0.005 for the others. The elasticity constant of the barrier is $\underline{k}_{b}=10^{7} \mathrm{~N} / \mathrm{m}$. The function $\mathbf{f}_{\mathrm{NL}}$ defined in Eq. (1) is then independent of the velocity and is written as

$$
f_{\mathrm{NL}}(y)= \begin{cases}0, & |y| \leq \varepsilon \\ -\underline{k}_{b}(y-\varepsilon \operatorname{sign}(y)), & |y|>\varepsilon\end{cases}
$$

\subsection{Mean model}

(A) Mean finite element model. The mean finite element model of the cantilever beam is constituted of 100 2-nodes Timoshenko beam elements. The first six computed eigenfrequencies are 26.9, 162.7, 432.9, 794.1, 1219.2 and $1685.3 \mathrm{~Hz}$.

(B) Description of excitation force. The vector load is defined by Eq. (2). The amplitude $a$ is considered as a parameter. The force is a point force applied at the middle point of the beam. The impulse function $g$ is such that

$$
g(t)=\frac{1}{\pi t}\left\{\sin \left(t\left(\Omega_{c}+\Delta \Omega / 2\right)\right)-\sin \left(t\left(\Omega_{c}-\Delta \Omega / 2\right)\right)\right\}
$$

whose Fourier Transform is $\widehat{g}(\omega)=\mathbf{1}_{\underline{B}_{2} \cup B_{2}}$. The frequency band $B_{2}$ is defined by Eq. (3) with $\omega_{\min }=2 \pi f_{\min }$ and $\omega_{\max }=2 \pi f_{\max }$ with $f_{\min }=148 \mathrm{~Hz}$ and $f_{\max }=178 \mathrm{~Hz}$. The corresponding bandwidth $\Delta \Omega=2 \pi \Delta f$ is then such that $\Delta f=30 \mathrm{~Hz}$ and the central frequency $\Omega_{c}=2 \pi f_{c}$ is such that $f_{c}=163$ Hz. Consequently, the frequency band of excitation is centered in the second 
eigenfrequency of the linear system.

(C) Reduced mean model. The numerical results presented in this section are computed with $n=40$, and the modes were calculated with the finite element model. This value was chosen to assure good convergence for the deterministic and the stochastic solutions.

\subsection{Probabilistic model of uncertainties}

(A) Parametric probabilistic model of the barrier. Since the gap is taken as a parameter of the problem it is not considered as uncertain. On the other hand, the stiffness of the barrier is uncertain and modelled by a positivevalued random variable $K_{b}$ whose mean value is $\underline{k}_{b}$, for which the coefficient of variation $\delta_{b}$ is 0 (no uncertainty) or 0.05 (uncertainty) and whose probability distribution is the Gamma law.

(B) Nonparametric probabilistic model of the beam. As explained in Section 3.3(B), the uncertainty levels for the mass, damping, and stiffness of the linear system are controlled by the dispersion parameters $\delta_{M}, \delta_{D}$, and $\delta_{K}$, respectively. In order to simplify the presentation, one only consider the cases $\delta_{M}=\delta_{D}=\delta_{K}$. The common valued will be denoted by $\delta_{\mathrm{m}}$. Two values are considered $\delta_{\mathrm{m}}=0$ (no uncertainty) and $\delta_{\mathrm{m}}=0.1$ (uncertainty).

\subsection{Numerical integration parameters}

Let $f_{0}>f_{\max }$ be the upper frequency such that the total energy of the response of the non-linear dynamical system is included in the frequency band $\left[-f_{0}, f_{0}\right]$. For all the numerical results presented below, a convergence analysis has been performed with respect to the value of $f_{0}$. The smallest value of $f_{0}$ for which all the results are converged is $f_{0}=600 \mathrm{~Hz}$ and all the results presented below correspond to this value of $f_{0}$. The integration time step is taken as $\Delta t=1 /\left(2 f_{0}\right)$ and the time integration $T=n_{\text {time }} \Delta t$ with $n_{\text {time }}=8192$. The integration in $\mathbb{R}$ is approximated by an integration over the interval $\left[t_{0}, t_{1}\right]$ in which $t_{0}=-T / 2$ and $t_{1}=T / 2-\Delta t$. The sampling time points are $t_{k}=t_{0}+$ 
$k \Delta t, k=0, \ldots, n_{\text {time }}-1$. To compute the Fourier Transform by FFT algorithm, the integration frequency step is taken as $\Delta \omega=2 \omega_{0} / n_{\text {freq }}$ with $n_{\text {freq }}=n_{\text {time. }}$. The sampling frequency points are $\omega_{k}=-\omega_{0}+k \Delta \omega, k=0, \ldots, n_{\text {freq }}-1$. Equation (31) is integrated over $\left[t_{0}, t_{1}\right]$ with zero initial conditions at $t_{0}$. The given choice of the parameters are such that $\mathcal{E}\left\{\left\|\mathbf{Q}\left(t_{1}, \theta\right)\right\|^{2}\right\}$ is negligible at the final time $t_{1}$.

\subsection{Numerical results}

Figure 1 displays the graph of the impulse function $t \mapsto g(t)$, whose Fourier Transform $f \mapsto \widehat{g}(2 \pi f)$ is shown in Fig. 2. Figure 3 displays the function $n_{s} \mapsto \operatorname{conv}\left(n_{s}, n\right)$ defined by Eq. (32) for $n=40$. The convergence is reached for $n_{s} \geq 1500$. Below, the results are computed with $n_{s}=2000$. Let $\eta=\varepsilon / a$ be the parameters that is used in the analysis of the random transferred energies outside the excitation band $B_{2}$. All the confidence regions shown in this work correspond to a probability level $P_{c}=0.96$.

The other results can be presented in several ways. To save space we show only one of the possible ways as an example. In the next section other forms to analyse the results are suggested. The example we have chosen is to present the results arranged by the values of the model and data uncertainties, $\left(\delta_{m}, \delta_{b}\right)$. This will give three blocks of results indexed by $(0.1,0.05),(0,0.05)$, and $(0.1,0)$. We present now the first block, the other two are similar. Figure 4 shows the random functions given by Eq. 25 that describe the random function energies in the frequency bands $B_{1}, B_{2}, B_{3}, B_{1} \cup B_{3}$ (figures 4 (a) to (d)). The mean system results are shown along with the mean of the stochastic system and the associated confidence limits. Figure 5 shows, for a fixed value of $\eta$ given by $\log _{10}(\eta)=-6.0$, the cumulative distribution functions of the energies. Figure 6 shows the density function defined by Eqs. 22 and 23 for three fixed values of $\eta$ such that $\log _{10}(\eta)=-6.0,-7.5,-4.6$, respectively (figures 6 (a) to (c)). Similar results (without cumulative distribution functions), for the case $(0,0.05)$ are shown in Figures 7 and 8 , and for the case $(0.1,0)$ are shown in Figures 9 and 10. 


\section{$5 \quad$ Analysis of the results and conclusions}

\subsection{Some remarks about the deterministic narrow-band excitation}

As explained in the introduction section, the excitation has been modelled by a deterministic narrow-band signal (see Figs. 1 and 2). Recall that the objective of the paper is to analyze the non-linear dynamical response of systems excited at a given resonance by a harmonic excitation. Since the mechanical system is uncertain, a narrow-band signal is chosen in order to give robustness to this excitation. Fig. 6 shows that the considered resonance of the uncertain system is effectively excited by the chosen narrow-band signal.

\subsection{Maximum of non-linearity effects as a function of $\eta$}

The measures of non-linearity is given by the fraction of energy that is transferred outside the band of excitation. Figures 4, 7 and 9 show that a maximum of non-linearity effect is obtained for mid-value of $\eta=\frac{\varepsilon}{a}$ and not for the extremes, near zero or very large. Near zero means that the gap is very small with respect to the displacement, that is there are a large number of impacts with low energy (small gap). This case is frequent, for instance, in Robotics (looseness). Very large means that the gap is sufficiently big with respect to the displacement such that the number of impacts is small and with low energy. In the medium range, the impacts are more frequent and also more energetic. It is worthwhile to insist that as $\eta$ is the ratio of the gap and the amplitude of the excitation, even for very small gaps the effect of $\eta$ can be large depending on the force. For example for $\varepsilon=2 \times 10^{-6} \mathrm{~m}$ a force of $1 N$ corresponds to a numerical value of $\eta=2 \times 10^{-6}$, and Fig. 4(d) shows that for this value there is a transfer of energy of 30 to 50 percent outside the band of excitation. 


\subsection{Relation of the non-linearities with the spectral density}

Figures 6(b), 8(b) and 10(b) (corresponding to $\eta=10^{-7.5}$ that is a near zero case) shows that although there is a small amount of energy transferred outside the band of excitation the effect of this transfer in the spectral density is very large causing the response to become a broad-band signal. Figures 6(c), 8(c) and (10c) (about $\eta=10^{-4.6}$ that is a very large case) shows that there is a very small amount of energy transferred outside the band of excitation so the system is nearly linear.

\section{$5.4 \quad$ Uncertainties effects}

Figure 4 shows that the point of maximum of non-linearities is also the point of less robustness with respect to uncertainties. On the other hand the two limit cases, near zero and very large $\eta$, are relatively robust with respect to uncertainties. Now one discusses the effect of two types of uncertainties: barrier uncertainties and model uncertainties for the continuous linear system. Figure 7 (corresponding to barrier uncertainties) and Fig. 9 (corresponding to model uncertainties) clearly show that the non-linearity effects are less robust for model uncertainties than for barrier uncertainties. This statement results from the following considerations. There is a loss of robustness induced by barrier uncertainties and by model uncertainties in the continuous linear subsystems (see Figs. 7(d) and 9(d)) in which the confidence region are significantly large. Comparing Fig. 4(d) with Fig. 9(d) shows that the two confidence regions are almost equal. This means that the effect of barrier uncertainties are less than the effects of model uncertainties. In addition, it can be seen that the confidence region in Fig. 7(d) is included (set inclusion) in the confidence region of Fig. 4(d). This result confirms the above statement. Also Figs. 6(a), $8(\mathrm{a})$ and 10(a) show that the maximum of non-linearity effects of the frequency response in the sub-harmonic and super-harmonic ranges is less robust for model uncertainties than for barrier uncertainties. On the other hand the frequency response in the frequency band of excitation is robust with respect to uncertainties. Finally, Fig. 5 allows the probability of the random energies 
to be estimated. For instance, Fig. 5(d) shows that the probability for that the percentage of energy transferred outside the band of excitation be larger than 20 percent is 0.75 and larger than 40 percent is 0.20 .

\subsection{Scope of the proposed method and its limitations}

It should be noted that the external excitation force is a narrow-band signal that is completely known. In this context, the proposed method is general to measure the non-linearity effects for the complete system. However, this method does not allow the non-linearity effects of its subsystems to be measured.

The probabilistic approach presented can be used to any system composed of a continuous linear subsystem (for instance, a Timoshenko beam) coupled with any discrete non-linear subsystem (for instance, an elastic barrier). Nevertheless, this kind of approach cannot be applied to non-linear continuous system such as an elastic system with large deformations. For such a case additional developments are required.

\section{Acknowledgments}

This work was supported by the Brazilian Agency Conselho Nacional de Desenvolvimento Científico e Tecnológico $(\mathrm{CNPq})$, by the International Cooperation Project Capes-Cofecub, $n^{\circ} 476 / 04$, and Faperj.

\section{References}

[1] V.I. Babitsky, N. Birkett, Theory of Vibro-impact Systems and Applications, Springer-Verlag, 1998.

[2] M.F. Dimentberg, A.A. Sokolov, Identification of restoring force non-linearity from system's response to a white-noise excitation, International Journal of Non-Linear Mechanics 26(6) (1991) 851-855.

[3] M.F. Dimentberg, D.V. Iourtchenko, Random vibrations with impacts: A review, Nonlinear Dynamics, 36 (2004) 229-254. 
[4] J.P. Cusumano, M.T. Sharkady, B.W. Kimble, Dynamics of a flexible beam impact oscillator, Philosophical Transactions of the Royal Society of London 347 (1994) 421-438.

[5] R. Bouc, M. Defilippi, Multimodal nonlinear spectral response of a beam with impact under random input, Probabilistic Engineerings Mechanics 12(3) (1997) 163-170.

[6] E. Emaci, T.A. Nayfeh, A.F. Vakakis, Numerical and experimental study of nonlinear localization in a flexible structure with vibro-impacts, Zeitschrift für Angewandte Mathematik und Mechanik (ZAMM) 77 (1997) 527-541.

[7] C. Wolter, M.A. Trindade, R. Sampaio, Reduced-order model for an impacting beam using the Karhunen-Loève expansion, Tendências em Matemática Aplicada e Computacional, TEMA 3(2) (2002) 217-226.

[8] M.A. Trindade, C. Wolter, R. Sampaio, Karhunen-Loève decomposition of coupled axial-bending vibrations of beams subject to impacts, Journal of Sound and Vibration 279 (3-5) (2005) 1015-1036.

[9] C. Soize, A nonparametric model of random uncertainties for reduced matrix model in structural dynamics, Probabilistic Engineering Mechanics 15(3) (2000) $277-294$.

[10] C. Soize, Random matrix theory for modeling uncertainties in computational mechanics, Computer Methods in Applied Mechanics and Engineering 194 (1216) (2005) 1333-1366.

[11] C.E. Shannon, A mathematical theory of communications, Bell System Technical Journal 27 (1948) 379-423.

[12] E.T. Jaynes, Information theory and statistical mechanics, Physical Review 106 (4) (1957) 620-630.

[13] E.T. Jaynes, Information theory and statistical mechanics, Physical Review 108 (2) (1957) 171-190. 


\section{Captions Accompanying Each Figure}

Fig. 1. Impulse function $t \mapsto g(t)$ exciting the system.

Fig. 2. Fourier Transform $f \mapsto \widehat{g}(2 \pi f)$ of the impulse function.

Fig. 3. Mean-square convergence: Function $n_{s} \mapsto \operatorname{conv}\left(n_{s}, n\right)$ for $n=40$.

Fig. $4 . \delta_{\mathrm{m}}=0.1$ and $\delta_{b}=0.05$ : Random fraction functions $\eta \mapsto E_{1}^{n}(\eta), E_{2}^{n}(\eta), E_{3}^{n}(\eta)$ and $E_{13}^{n}(\eta)$ related to the random mechanical energy transferred to band $B_{1}$ (fig. (a)), $B_{2}$ (fig. (b)), $B_{3}$ (fig. (c)) and $B_{1} \cup B_{3}$ (fig. (d)). Mean system (thin solid line). Mean value for the stochastic system (thick solid line). Confidence region (grey region).

Fig. 5. $\delta_{\mathrm{m}}=0.1$ and $\delta_{b}=0.05$ : For $\log _{10} \eta=-6.0$, cumulative distribution function $\zeta^{1} \mapsto \operatorname{Proba}\left\{E_{1}^{n}(\eta) \leq \zeta^{1}\right\}$ (fig. (a)), $\zeta^{2} \mapsto \operatorname{Proba}\left\{E_{2}^{n}(\eta) \leq \zeta^{2}\right\}$ (fig. (b)), $\zeta^{3} \mapsto \operatorname{Proba}\left\{E_{3}^{n}(\eta) \leq \zeta^{3}\right\}$ (fig. (c)) and $\zeta^{13} \mapsto \operatorname{Proba}\left\{E_{13}^{n}(\eta) \leq \zeta^{13}\right\}$ (fig. (d)) related to the random mechanical energy transferred to band $B_{1}, B_{2}, B_{3}$ and $B_{1} \cup B_{3}$.

Fig. 6. $\delta_{\mathrm{m}}=0.1$ and $\delta_{b}=0.05$ : For $\log _{10} \eta=-6.0$ (fig. (a)), $\log _{10} \eta=-7.5$ (fig. (b)) and $\log _{10} \eta=-4.6$ (fig. (c)), graphs of the random normalized energy density $f \mapsto H_{\mathrm{dB}}^{n}(2 \pi f)$. Mean system (thin solid line). Mean value for the stochastic system (thick solid line). Confidence region (grey region).

Fig. 7. $\delta_{\mathrm{m}}=0$ and $\delta_{b}=0.05$ : Random fraction functions $\eta \mapsto E_{1}^{n}(\eta), E_{2}^{n}(\eta), E_{3}^{n}(\eta)$ and $E_{13}^{n}(\eta)$ related to the random mechanical energy transferred to band $B_{1}$ (fig. (a)), $B_{2}$ (fig. (b)), $B_{3}$ (fig. (c)) and $B_{1} \cup B_{3}$ (fig. (d)). Mean system (thin solid line). Mean value for the stochastic system (thick solid line). Confidence region (grey region).

Fig. 8. $\delta_{\mathrm{m}}=0$ and $\delta_{b}=0.05$ : For $\log _{10} \eta=-6.0$ (fig. (a)), $\log _{10} \eta=-7.5$ (fig. (b)) and $\log _{10} \eta=-4.6$ (fig. (c)), graphs of the random normalized energy density $f \mapsto H_{\mathrm{dB}}^{n}(2 \pi f)$. Mean system (thin solid line). Mean value for the stochastic system (thick solid line). Confidence region (grey region).

Fig. 9. $\delta_{\mathrm{m}}=0.1$ and $\delta_{b}=0$ : Random fraction functions $\eta \mapsto E_{1}^{n}(\eta), E_{2}^{n}(\eta), E_{3}^{n}(\eta)$ and $E_{13}^{n}(\eta)$ related to the random mechanical energy transferred to band $B_{1}$ 
(fig. (a)), $B_{2}$ (fig. (b)), $B_{3}$ (fig. (c)) and $B_{1} \cup B_{3}$ (fig. (d)). Mean system (thin solid line). Mean value for the stochastic system (thick solid line). Confidence region (grey region).

Fig. 10. $\delta_{\mathrm{m}}=0.1$ and $\delta_{b}=0$ : For $\log _{10} \eta=-6.0$ (fig. (a)), $\log _{10} \eta=-7.5$ (fig. (b)) and $\log _{10} \eta=-4.6$ (fig. (c)), graphs of the random normalized energy density $f \mapsto H_{\mathrm{dB}}^{n}(2 \pi f)$. Mean system (thin solid line). Mean value for the stochastic system (thick solid line). Confidence region (grey region). 


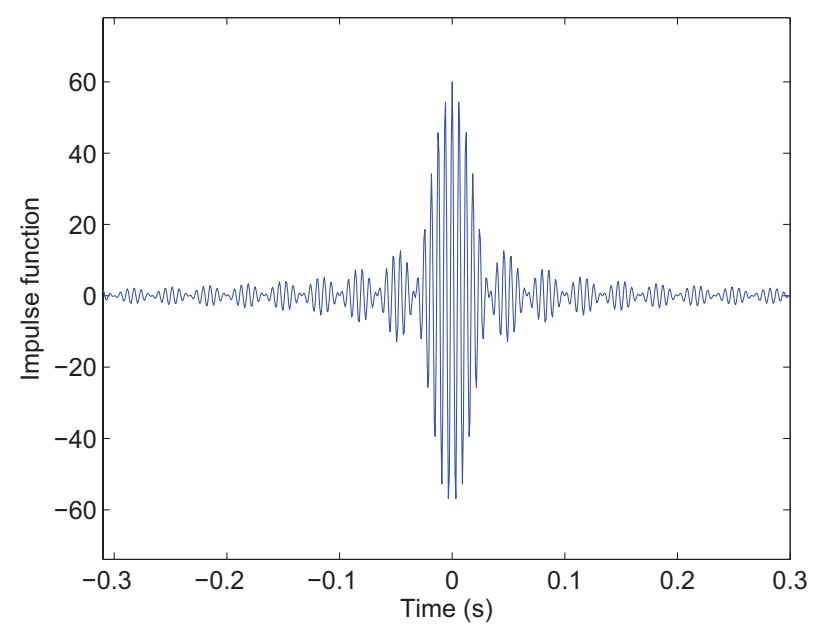

Fig. 1.

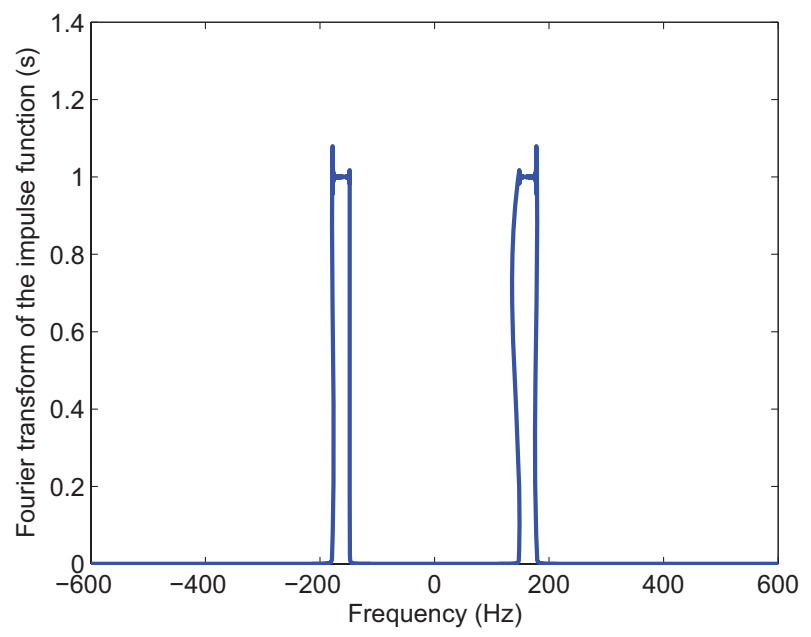

Fig. 2. 


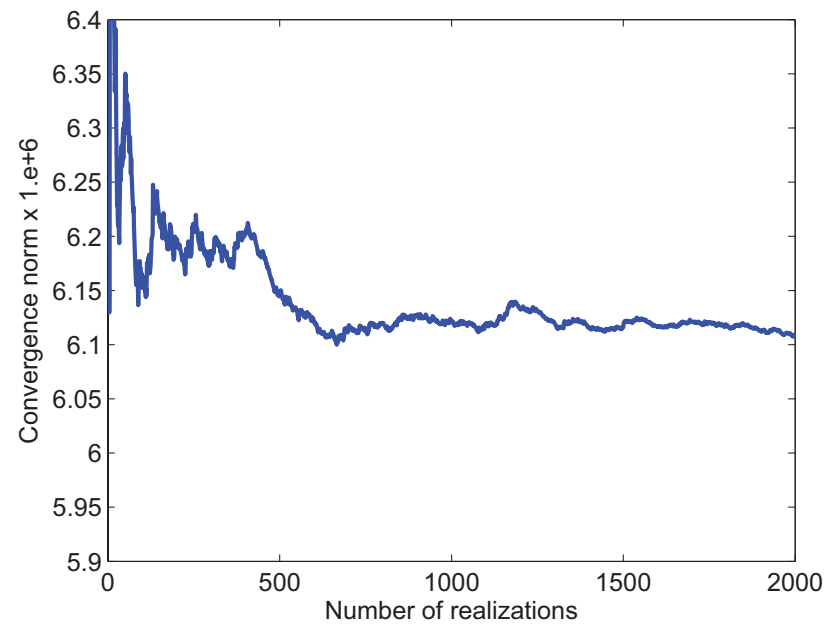

Fig. 3.
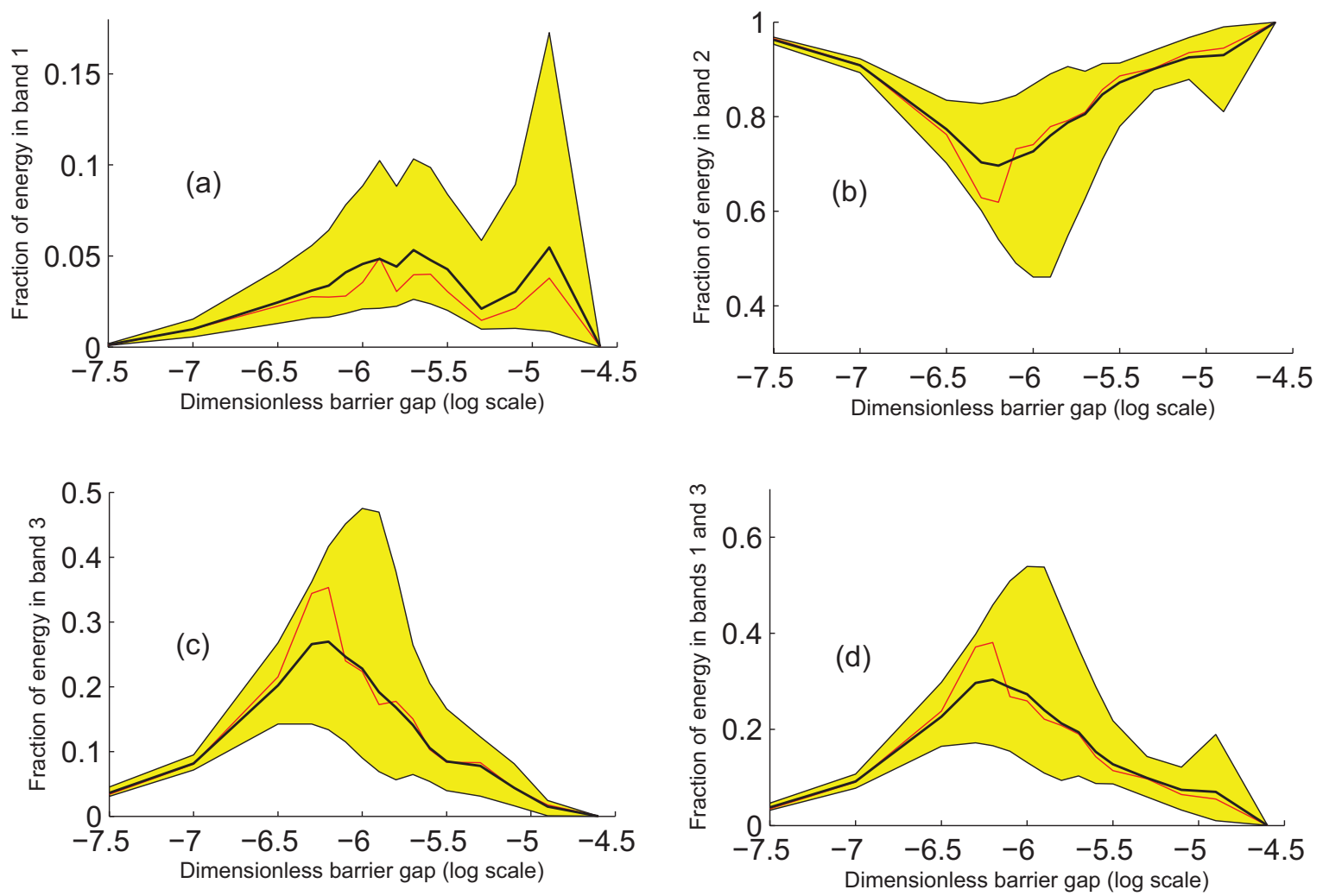

Fig. 4. 

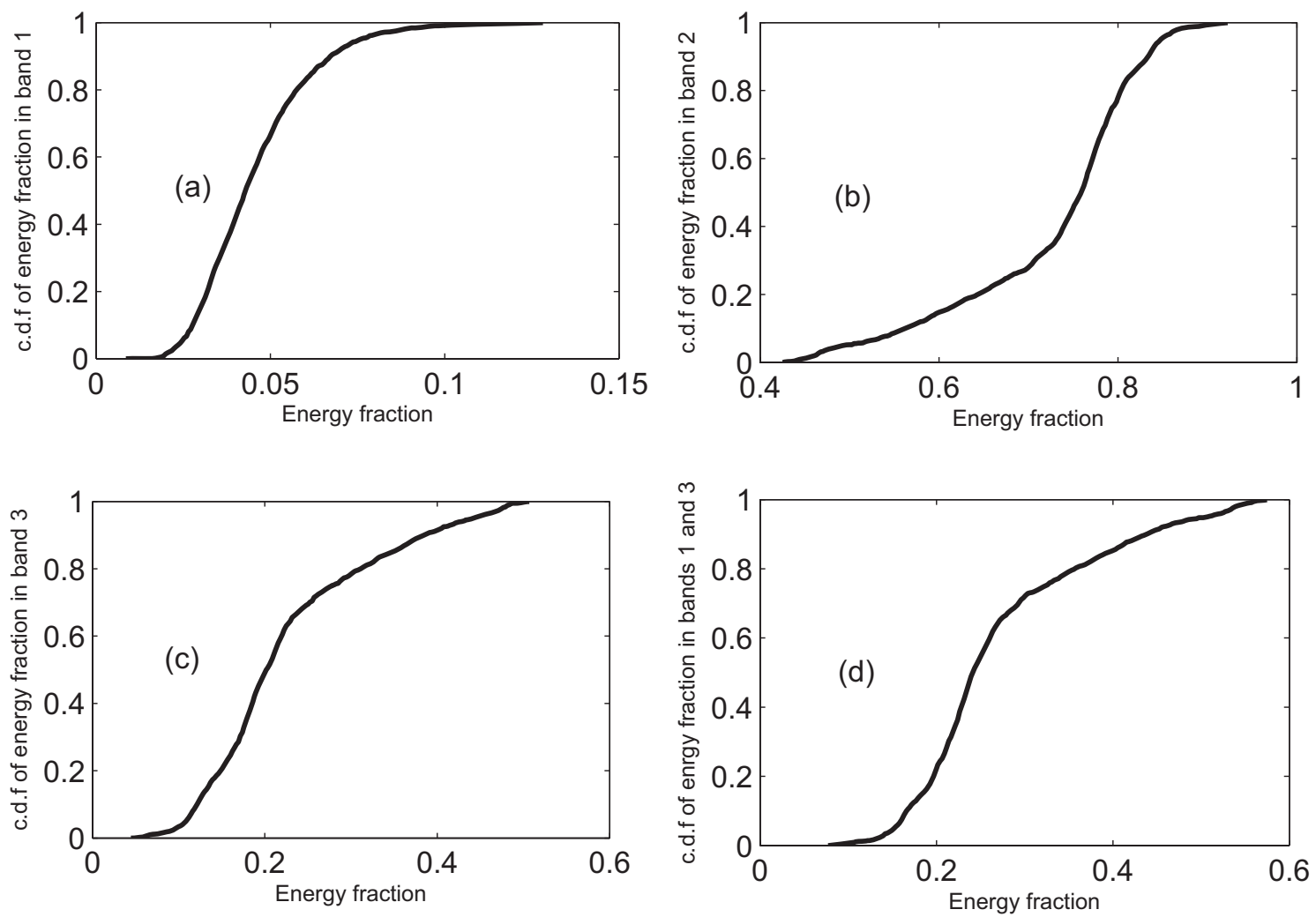

Fig. 5. 

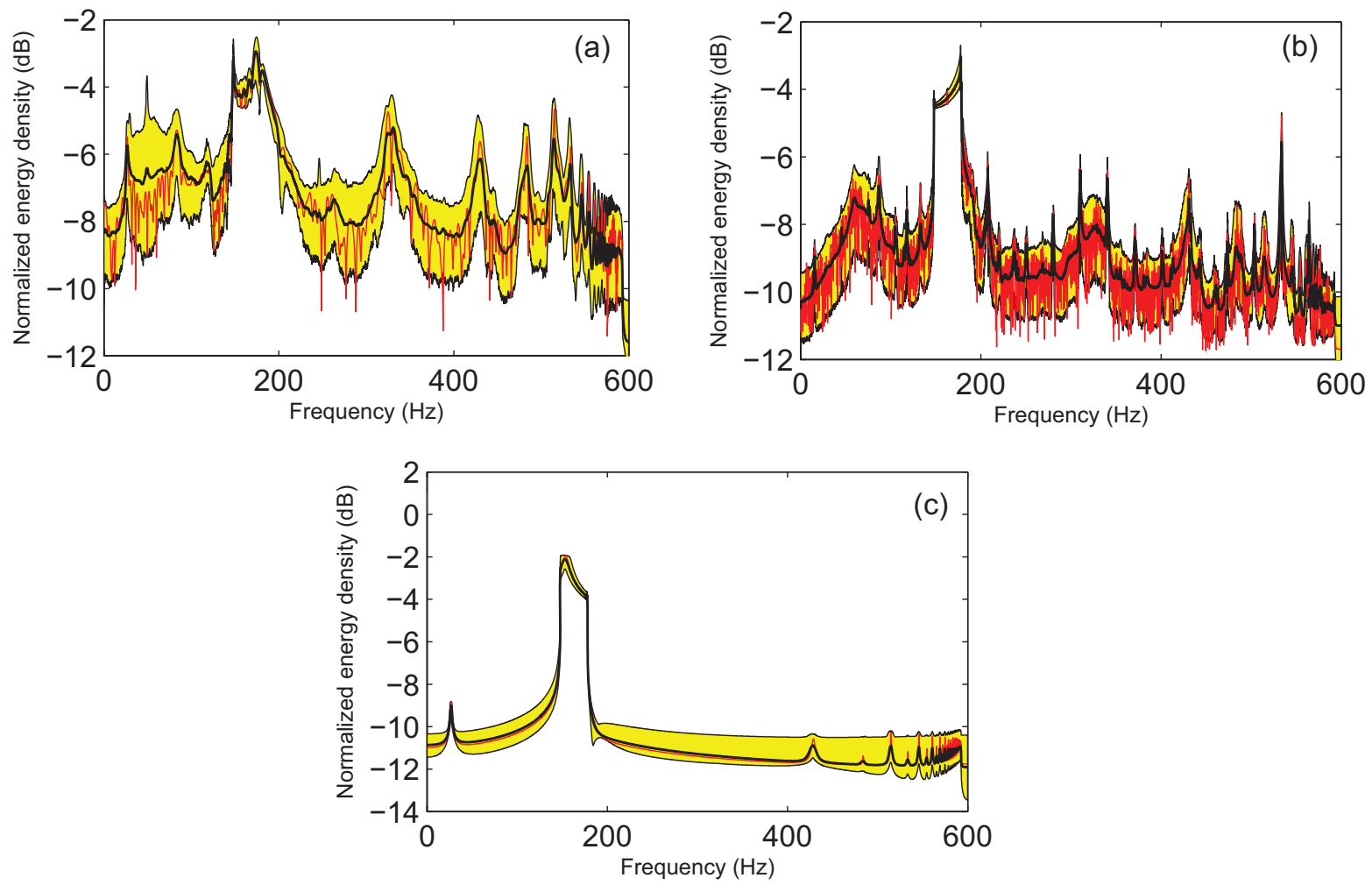

Fig. 6 . 

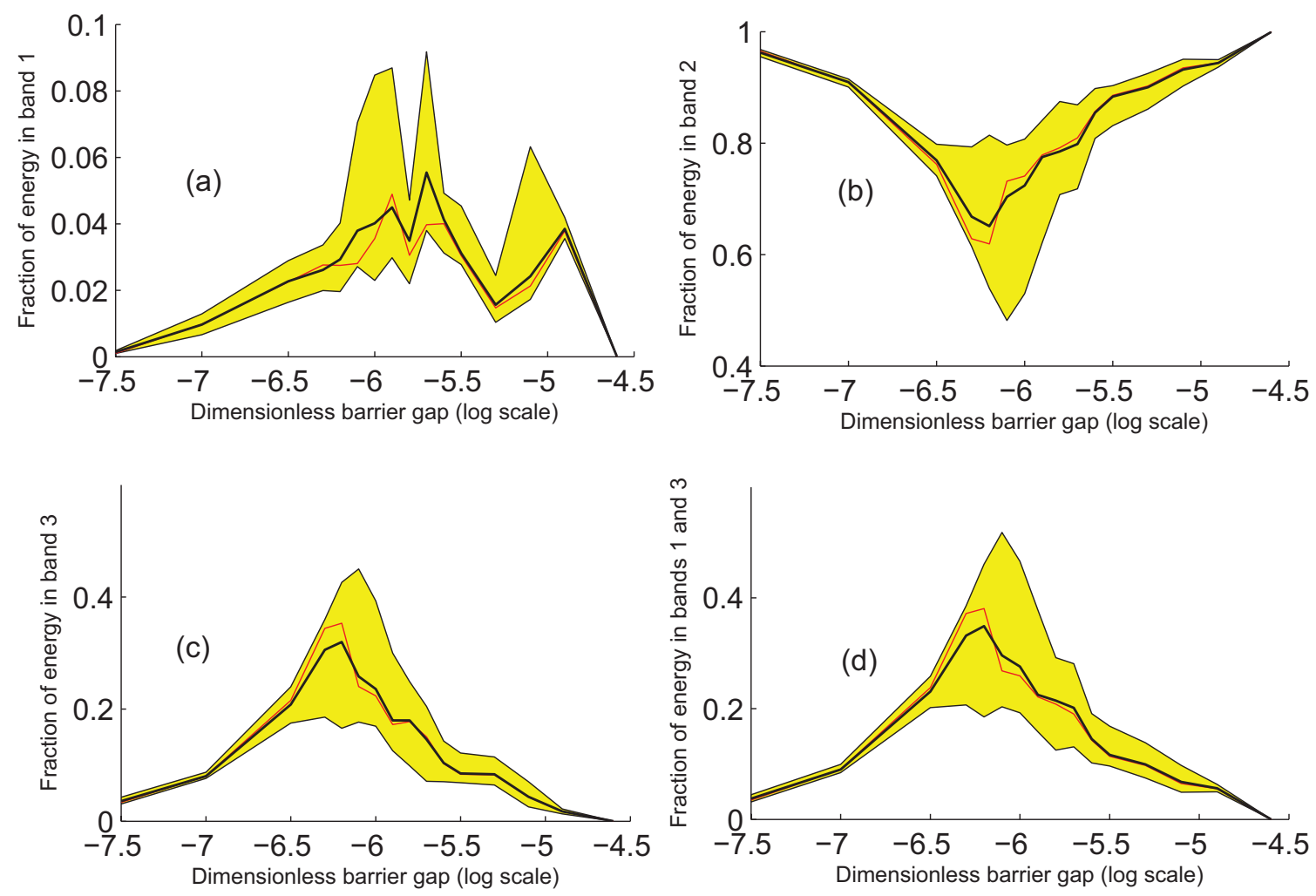

Fig. 7. 

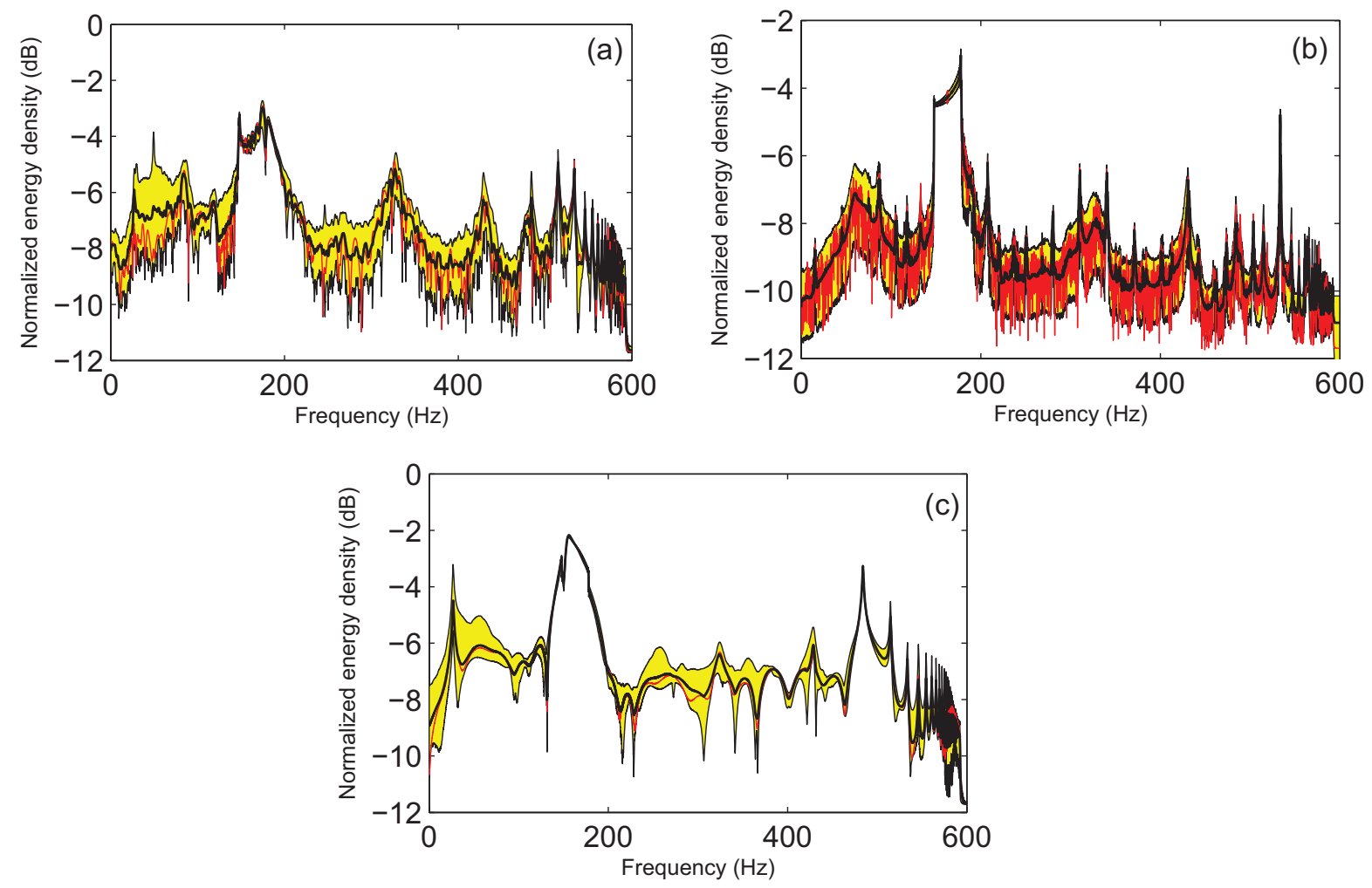

Fig. 8. 

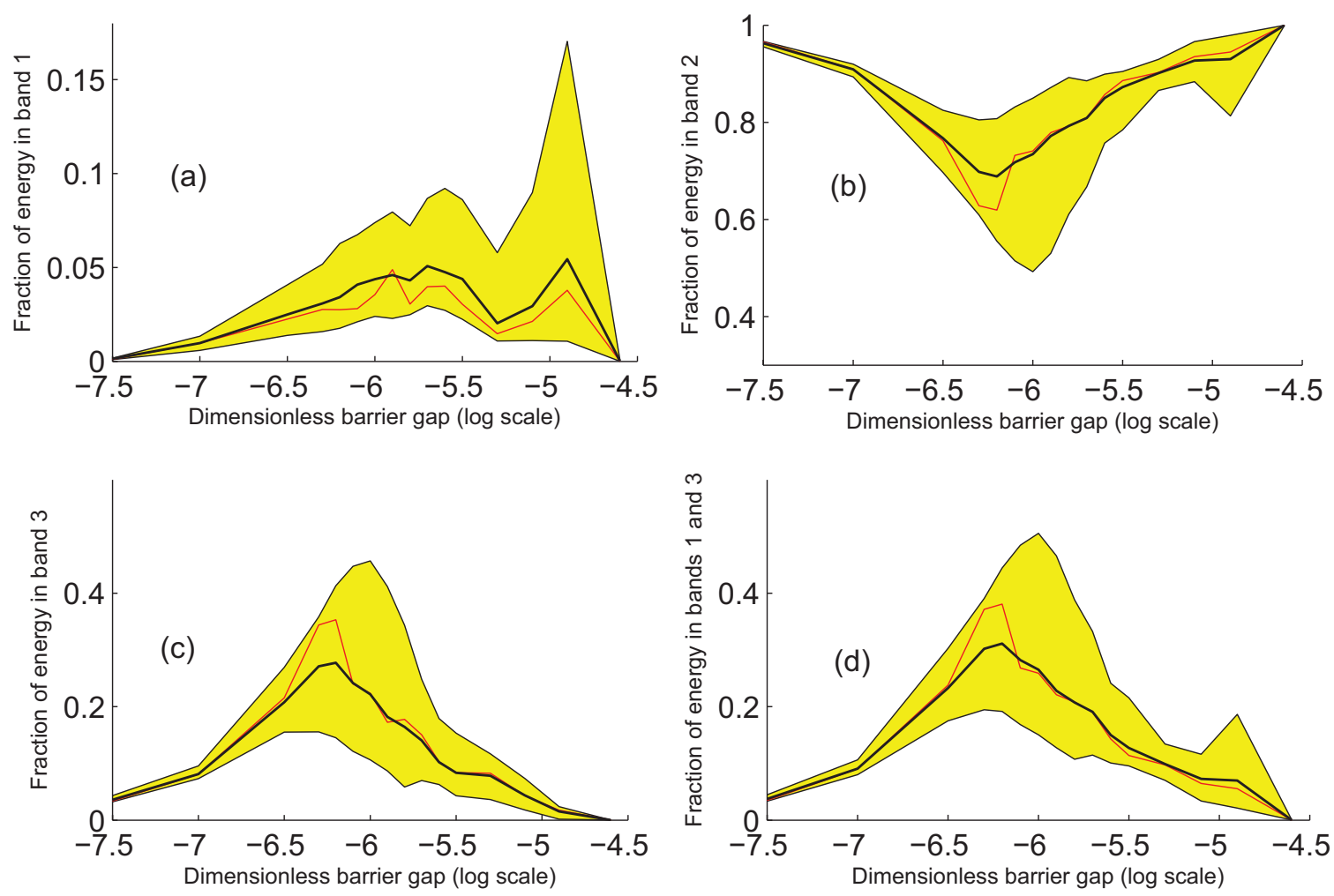

Fig. 9. 

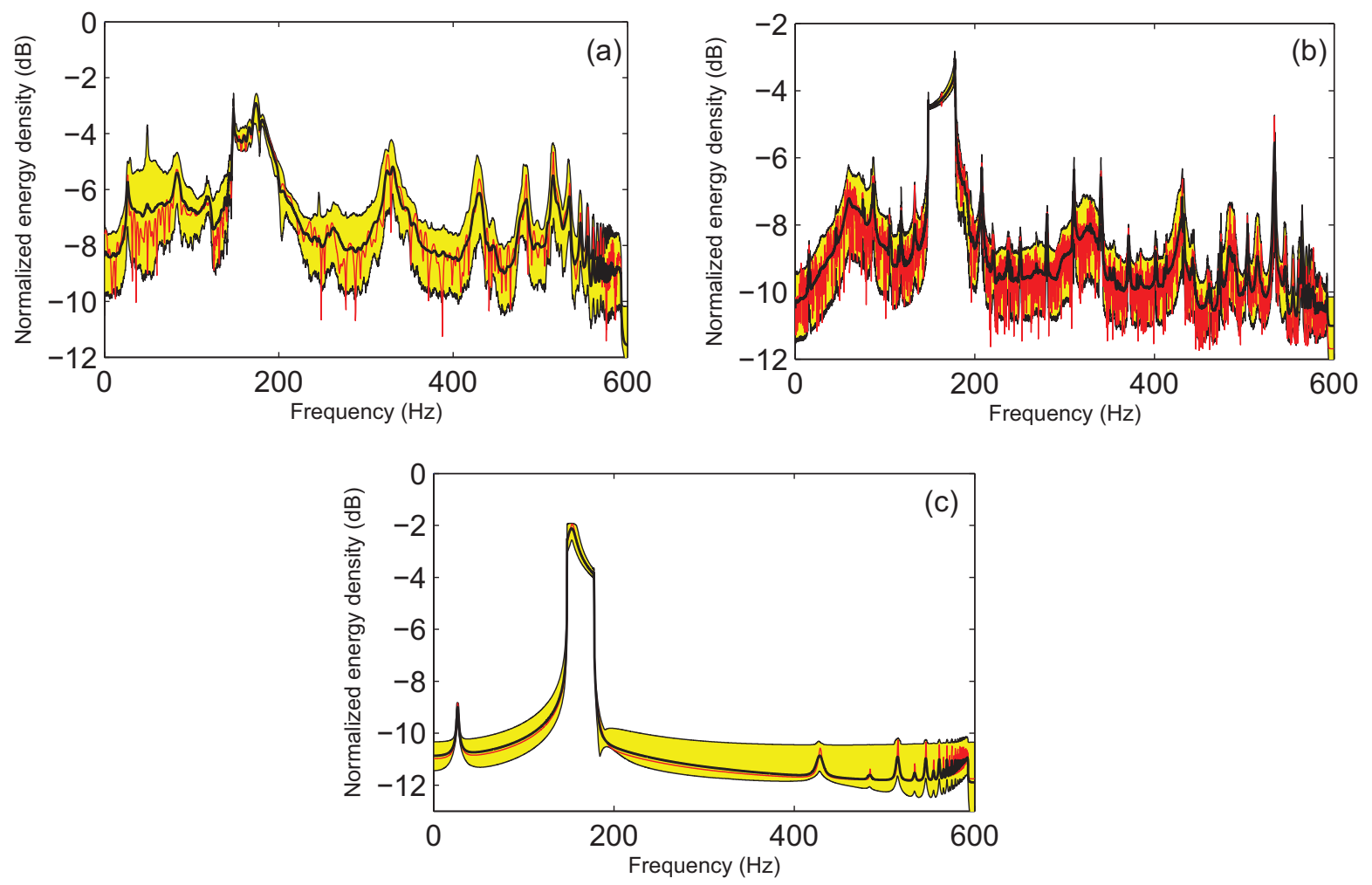

Fig. 10. 